\title{
FLUCTUATING BOUNDARY LAYERS IN A ROTATING FLUID WITH VARIABLE SUCTION*
}

\author{
BY R. PURUSHOTHAMAN (Indian Institute of Technology, Kharagpur)
}

\begin{abstract}
The viscous flow in a semi-infinite fluid above a porous oscillating disk with unsteady normal velocity in a rotating frame is studied. Expressions for velocity and skin friction are obtained. For the case of suction, the oscillations are contained in boundary layers of finite thickness. In the case of injection, resonance occurs when the imposed frequency is twice the angular velocity of rotation. It is found that the resonance can be avoided under certain conditions.
\end{abstract}

1. Introduction. The flow generated in a semi-infinite fluid by a nontorsionally oscillating disk in a rotating frame has been studied in detail by Thornley [1]. Modified Stokes layers are formed on the disk for all frequencies, except for the resonance frequency which is twice the angular velocity of rotation $\Omega$. In such a case there is no oscillatory solution which can satisfy all the boundary conditions. Thornley [1] proves rigorously that this resonance problem can be resolved if (i) the velocity vector of the disk rotates with constant magnitude as the basic angular rotation or (ii) another disk is placed parallel to and at some finite distance $d$ away from the first disk.

The present paper is devoted to a study of the effects of unsteady suction or blowing on the unsteady boundary layer flow in an incompressible homogeneous viscous fluid in a rotating frame. The normal velocity at the disk is taken as $w_{0}(1+\epsilon A \cos \omega t)$. The velocity components $u$ and $v$ in the plane of the disk are given by

$$
u+i v=U[c+\epsilon(a \exp (i \omega t)+b \exp (-i \omega t))],
$$

where $U$ is constant with the dimensions of velocity and $a, b, c$ are complex constants. The method used by Kelly [2] for the solution of a similar problem in the non-rotating case is adopted. A Fourier expansion for the velocity is made which results in an infinite set of coupled equations. Assuming $\epsilon<1$, an expansion is performed in powers of $\epsilon$ and the equations thus obtained are solved. It is found that due to the presence of fluctuating normal velocity a series of oscillatory boundary layers evolve, corresponding to different modes of oscillation. All the boundary layers are bounded and a meaningful solution exists in the case of suction. On the contrary, in the presence of blowing, the resonance behavior persists when $n \omega \rightarrow 2 \Omega, n=1,2,3, \ldots$ A necessary condition for obtaining a meaningful solution in this case is given. For all other frequencies the oscillations are confined to boundary layers of finite thickness.

Stuart [3] gave an exact solution for the oscillatory flow over an infinite plate with constant suction. Kelly [2] and Messiha (4) subsequently extended Stuart's work by imposing unsteady suction at the boundary. In a recent paper Purushothaman [5] generalized the situation considered by Messiha [4] by including the effect of Coriolis force with

* Received July 19. 1977. The author expresses his thanks to Dr. S. S. Chawla for valuable suggestions and guidance. 
$\epsilon<<1$. The results of Purushothaman [5] can be obtained as a particular case of the present analysis.

2. Mathematical formulation. A semi-infinite expanse of an incompressible viscous fluid is bounded by an infinite disk at $z=0$. Both the fluid and the disk rotate as a solid body with constant angular velocity $\Omega$ about an axis normal to the disk. We study the effect of an unsteady normal velocity at the disk on the fluctuating flow due to a timedependent motion of the disk. We take cartesian coordinates $(x, y, z)$ such that the $z$-axis is parallel to the axis of rotation and the $x$ and $y$ axes lie on the plane of the disk. The unsteady motion of the fluid in this rotating coordinate system is governed by the equations

$$
\begin{gathered}
\frac{D \mathbf{V}}{D t}+2 \Omega \times \mathbf{V}=-\frac{1}{\rho} \operatorname{grad} p+\nu \nabla^{2} \mathbf{V}, \\
\operatorname{div} \mathbf{V}=0,
\end{gathered}
$$

where $\mathbf{V}=(u, v, w)$ is the velocity vector, $p$ is the effective kinematic pressure, $\rho$ is the density and $\nu$ is the kinematic viscosity of the fluid.

We assume that the velocity field is independent of $x$ and $y$. The boundary conditions are taken as

$$
\begin{aligned}
u+i v & =U[c+\epsilon(a \exp (i \omega t)+b \exp (-i \omega t))], \text { on } z=0 \\
w & =w_{0}(1+\epsilon A \cos \omega t), u, v \rightarrow 0 \quad \text { as } z \rightarrow \infty,
\end{aligned}
$$

where $a, b, c$ are complex constants, $A$ is a suction parameter and $\epsilon$ is a small positive constant. Obviously $w_{0}<0$ gives suctions and $w_{0}>0$ gives injection. From the equation of continuity, it follows immediately that

$$
w=w_{0}(1+\epsilon A \cos \omega t) .
$$

Then the equations of motion take the form

$$
\begin{gathered}
\frac{\partial u}{\partial t}+w_{0}(1+\epsilon A \cos \omega t) \frac{\partial u}{\partial z}-2 \Omega v=-\frac{1}{\rho} \frac{\partial p}{\partial x}+\nu \frac{\partial^{2} u}{\partial z^{2}}, \\
\frac{\partial v}{\partial t}+w_{0}(1+\epsilon A \cos \omega t) \frac{\partial v}{\partial z}+2 \Omega u=-\frac{1}{\rho} \frac{\partial p}{\partial y}+\nu \frac{\partial^{2} v}{\partial z^{2}}, \\
\frac{\partial w}{\partial t}=-\frac{1}{\rho} \frac{\partial p}{\partial z} .
\end{gathered}
$$

As there is no relative motion far from the disk, we assume that there is no imposed pressure gradient along the $x$ and $y$ directions, i.e.

$$
\partial p / \partial x=0, \quad \partial p / \partial y=0,
$$

and the pressure gradient normal to the disk is given by (7).

With the complex notation

$$
q^{\prime}=u+i v
$$

the equations (5) and (6) become

$$
\frac{\partial q^{\prime}}{\partial t}+w_{0}(1+\epsilon A \cos \omega t) \frac{\partial q^{\prime}}{\partial t}+2 \Omega i q^{\prime}=\nu \frac{\partial^{2} q^{\prime}}{\partial z^{2}},
$$


and the boundary conditions become

$$
\begin{aligned}
q^{\prime}(0, t) & =U[c+\epsilon(a \exp (i \omega t)+b \exp (-i \omega t))], \\
q^{\prime}(\infty, t) & =0 .
\end{aligned}
$$

The equation (10) is non-dimensionalized by the transformations

$$
\begin{array}{ll}
q=q^{\prime} / U, & \eta=z\left|w_{0}\right| / \nu, \\
\tau=w_{0}^{2} t / 4 \nu, & \alpha=4 \nu \omega / w_{0}{ }^{2}, \\
E=\nu \Omega / w_{0}^{2}, & S=w_{0} /\left|w_{0}\right| .
\end{array}
$$

Thus we obtain

$$
\frac{\partial^{2} q}{\partial \eta^{2}}-S\left[1+\frac{\epsilon A}{2}(\exp (i \alpha \tau)+\exp (-i \alpha \tau))\right] \frac{\partial q}{\partial \eta}-2 E i q-\frac{1}{4} \frac{\partial q}{\partial \tau}=0,
$$

with

$$
\begin{aligned}
q(0, \tau) & =c+\epsilon[a \exp (i \alpha \tau)+b \exp (-i \alpha \tau)] \\
q(\infty, \tau) & =0
\end{aligned}
$$

3. Solution of the problem. In order to solve (13) subjected to (14), we assume

$$
q(\eta, \tau)=\sum_{-\infty}^{\infty} q_{n}(\eta) \exp (i n \alpha \tau)
$$

Substituting (15) in (13) and (14), we obtain the equation for $q_{n}$ as

$$
\frac{d^{2} q_{n}}{d \eta^{2}}-S\left[\frac{d q_{n}}{d \eta}+\frac{\epsilon A}{2}\left(\frac{d q_{n-1}}{d \eta}+\frac{d q_{n+1}}{d \eta}\right)\right]-i(2 E+n \alpha / 4) q_{n}=0,
$$

with the boundary conditions

$$
\begin{aligned}
q_{0}(0)=c, & q_{1}(0)=a, & q_{-1}(0)=b, \\
q_{n}(0)=0, & |n|>1, & q_{n}(\infty)=0 .
\end{aligned}
$$

The coupled system of differential equations (16) is solved assuming $\epsilon<1$. For this we expand $q_{n}$ in a series of the form

$$
q_{n}=\sum_{j=0}^{\infty} \epsilon^{j} q_{n j}(\eta)
$$

Substituting (18) in (16) and equating the same powers of $\epsilon$, we obtain the following differential equation for $q_{n j}$ :

$$
q_{n j}^{\prime \prime}-S q_{n j}^{\prime}-i(2 E+n \alpha / 4) q_{n j}=-\frac{A}{2}\left(q_{n-1, j-1}^{\prime}+q_{n+1, j-1}^{\prime}\right) .
$$

Here a prime denotes a differentiation with respect to $\eta$. The boundary conditions for $q_{n j}$ are

$$
\begin{gathered}
q_{00}(0)=c, \quad q_{11}(0)=a, \quad q_{-11}(9)=b, \\
q_{n j}(0)=0 \text { for all other } n \text { and } j, q_{n j}(\infty)=0 \text { for all } n \text { and } j .
\end{gathered}
$$


The solutions are

$$
\begin{gathered}
q_{00}=c \exp \left(m_{0} \eta\right), \\
q_{n 0}=0, \quad n \neq 0, \\
q_{11}=a \exp \left(m_{1} \eta\right)+\frac{2 A \operatorname{cim}_{0}}{\alpha}\left(\exp \left(m_{1} \eta\right)-\exp \left(m_{0} \eta\right)\right), \\
q_{-11}=l e \exp \left(m_{2} \eta\right)-\frac{2 A c i m_{0}}{\alpha}\left(\exp \left(m_{2} \eta\right)-\exp \left(m_{0} \eta\right),\right. \\
q_{n 1}=0, \quad n \neq-1,1, \\
q_{02}=\frac{2 A i}{\alpha}\left\{\operatorname { a m } _ { 1 } \left(\exp \left(m_{1} \eta\right)-\exp \left(m_{0} \eta\right)-b m_{2}\left(\exp \left(m_{2} \eta\right)-\exp \left(m_{0} \eta\right)\right.\right.\right. \\
+\frac{2 A \operatorname{cim}_{0}}{\alpha}\left[m_{1} \exp \left(m_{1} \eta\right)+m_{2} \exp \left(m_{2} \eta\right)-\left(m_{1}+m_{2}\right) \exp \left(m_{0} \eta\right]\right\}, \\
q_{22}=\frac{2 A_{i}}{\alpha}\left\{\operatorname{am}_{1}\left(\exp \left(m_{1} \eta\right)-\exp \left(m_{3} \eta\right)\right)\right. \\
+\frac{A \operatorname{cim}_{0}}{\alpha}\left[2 m_{1}\left(\exp \left(m_{1} \eta\right)-\exp \left(m_{2} \eta\right)\right)+\frac{m_{0}}{\alpha}\left(\exp \left(m_{0} \eta\right)-\exp \left(m_{2} \eta\right)\right]\right\},
\end{gathered}
$$

where

$$
\begin{gathered}
m_{0}=\frac{1}{2}\left\{S-\left(S^{2}+8 i E\right)^{1 / 2}\right\}, \\
m_{1}=\frac{1}{2}\left\{S-\left[S^{2}+i(8 E+\alpha)\right]^{1 / 2}\right\}, \\
m_{2}=\frac{1}{2}\left\{S-\left[S^{2}+i(8 E-\alpha)\right]^{1 / 2}\right\}, \\
m_{3}=\frac{1}{2}\left\{S-\left[S^{2}+i(8 E+2 \alpha)\right]^{1 / 2}\right\}, \text { etc. }
\end{gathered}
$$

The shear stresses $\tau_{x}$ and $\tau_{y}$ at the disk are given by

$$
\begin{aligned}
\left(\tau_{x}+i \tau_{y}\right) / \rho u\left|w_{0}\right|= & c m_{0}+\epsilon \exp (i \alpha \tau)\left[a m_{1}+\frac{2 A \operatorname{cim}_{0}}{\alpha}\left(m_{1}-m_{0}\right)\right] \\
& +\epsilon \exp (-i \alpha \tau)\left[\text { lem }_{2}-\frac{2 A \operatorname{cim}_{0}}{\alpha}\left(m_{2}-m_{0}\right)\right]+O\left(\epsilon^{2}\right) .
\end{aligned}
$$

4. Discussion of the results. The solution given by (21)-(27) describes the general features of the unsteady boundary layers in a rotating system. The solution reduces to the quasi-steady-state solution of Debnath and Mukherjee [6] when $A=0$. Setting $E=0$, we can retrieve the results of Kelly [2] and Messiha [4] for the similar problem in the nonrotating frame. The results of Purushothaman (5) can be obtained by putting $a=1, b=0$, $c=1$ and $S=-1$. We see that a series of boundary layers evolve because of the oscillations. The thickness of various boundary layers is of $O\left(\delta_{n}\right)$ where

$$
\delta_{n}=\text { real part of }\left(\nu /\left|w_{0}\right| m_{n}\right) .
$$

Also we note that $q_{n} \sim O\left(\epsilon^{|n|}\right)$. When $c=0$ and $a \neq 0, b \neq 0$, the disk performs an elliptic harmonic motion and the Ekman layer (duly modified by suction) appears only in the 
$O\left(\epsilon^{2}\right)$ term. When $\alpha=0, m_{0}=m_{1}=m_{2}=\cdots$ and hence all the boundary layers coalesce into a single modified Ekman layer of thickness $\delta_{0}$.

In the case of suction $S=-1$ and the oscillations are confined to the boundary layers for all frequencies. In the absence of suction a resonance behavior was predicted by Thornley [1] for $\omega=2 \Omega$. But here we note that no such behavior is present when $\omega=2 \Omega$, i.e. $8 E=\alpha$. One of the boundary layers has a thickness of $O\left(\nu /\left|w_{0}\right|\right)$ which is independent of rotation and depends on the suction. As can be expected, the boundary layer thicknesses, in general, decrease due to the increase of suction.

For the ease of blowing, $S=1$ and the boundary layers are bounded except when $8 E=$ $\alpha, 2 \alpha, \alpha, \cdots$ i.e. $\omega=2 \Omega, \Omega, 2 \Omega / 3, \cdots$. For $8 E=n \alpha$, one of the boundary layers becomes infinitely deep. To order $\epsilon$, the resonance behaviour occurs when $\omega=2 \Omega$, in which case the function $q_{-11}$ has the form

$$
q_{-11}=b-\frac{2 A \operatorname{cim}_{0}}{\alpha}\left(1-\exp \left(m_{0} \eta\right)\right),
$$

and $m_{2}=0$. This solution satisfies the correct boundary condition at the disk and is finite but non-zero as $\eta \rightarrow \infty$. A solution satisfying all the correct boundary conditions cannot be found except when

$$
b=2 A \operatorname{cim}_{0} / \alpha .
$$

When the injection is constant or the disk performs pure oscillations this condition becomes $b=0$.

Finally, we consider the inviscid region performing oscillations instead of the disk. Then let

$$
\begin{gathered}
u(\infty, t)+i v(\infty, t)=q^{*}(\infty, t)=-q^{\prime}(0, t), \\
q^{*}(0, t)=0 .
\end{gathered}
$$

Due to inviscid oscillations, pressure gradients are non-zero and hence, using

$$
\frac{\partial p^{*}}{\partial x}+i \frac{\partial p^{*}}{\partial y}=P
$$

we obtain the equation of motion as

$$
\frac{\partial q^{*}}{\partial t}+w_{0}(1+\epsilon A \cos \omega t) \frac{\partial q^{*}}{\partial z}+2 \Omega i q^{*}=-P+\nu \frac{\partial^{2} q^{*}}{\partial z^{2}} .
$$

Then $P$ is given by

$$
\begin{gathered}
P=-\left[\frac{\partial q^{*}}{\partial t}(\infty, t)+2 \Omega i q^{*}(\infty, t)\right] \\
=U[2 \Omega i c+i \epsilon\{a(\omega+2 \Omega) \exp (i \omega t)+b(2 \Omega-\omega) \exp (-i \omega t)\}] .
\end{gathered}
$$

Setting

$$
Q(z, t)=q^{*}(z, t)-q^{*}(\infty, t)
$$

we arrive at the equation

$$
\frac{\partial Q}{\partial t}+w_{0}(1+\epsilon A \cos \omega t) \frac{\partial Q}{\partial z}+2 \Omega i Q=\nu \frac{\partial^{2} Q}{\partial z^{2}},
$$


with the boundary conditions

$$
Q(0, t)=q^{\prime}(0, t), \quad Q(\infty, t)=0 .
$$

These are the same as Eqs. (10) and (11) whose solution has been discussed.

\section{REFERENCES}

[1] C. Thornley, Quart. J. Mech. Appl. Math. 21, 451 (1968)

[2] R. E. Kelly, Quart. J. Mech. Appl. Math. 28, 287 (1965)

[3] J. T. Stuart, Proc. Roy. Soc. London. A 231, 116 (1955)

[4] S. A. S. Messiha, Proc. Camb. Phil. Soc. 62, 329 (1966)

[5] R. Purushothaman, J. Inst. Math. Appl. 20, 257 (1977)

[6] L. Debnath and S. Mukherjee, Phys. Fluids 16, 1418 (1973) 